\title{
A quantum annealer with fully programmable all-to-all coupling via Floquet engineering
}

\author{
Tatsuhiro Onodera (iD) ${ }^{1,2,3,4}$, Edwin Ng iD $^{2,4}$ and Peter L. McMahon (iD $^{1,2 凶}$
}

Quantum annealing is a promising approach to heuristically solving difficult combinatorial optimization problems. However, the connectivity limitations in current devices lead to an exponential degradation of performance on general problems. We propose an architecture for a quantum annealer that achieves full connectivity and full programmability while using a number of physical resources only linear in the number of spins. We do so by application of carefully engineered periodic modulations of oscillatorbased qubits, resulting in a Floquet Hamiltonian in which all the interactions are tunable. This flexibility comes at the cost of the coupling strengths between qubits being smaller than they would be compared with direct coupling, which increases the demand on coherence times with increasing problem size. We analyze a specific hardware proposal of our architecture based on Josephson parametric oscillators. Our results show how the minimum-coherence-time requirements imposed by our scheme scale, and we find that the requirements are not prohibitive for fully connected problems with up to at least 1000 spins. Our approach could also have impact beyond quantum annealing, since it readily extends to bosonic quantum simulators, and would allow the study of models with arbitrary connectivity between lattice sites.

npj Quantum Information (2020)6:48; https://doi.org/10.1038/s41534-020-0279-z

\section{INTRODUCTION}

Quantum annealers are computational devices designed for solving combinatorial optimization problems, most typically Ising optimization problems ${ }^{1-3}$. An Ising problem is specified by connections among $N$ spins on a graph, as well as local fields on each spin. One of the foremost challenges in the experimental realization of quantum annealers is the requirement that quantum annealers be able to represent densely connected Ising problems with minimal overhead in the number of qubits (and other physical components) used ${ }^{4-7}$. If a quantum annealer is not able to directly represent a particular problem because the problem graph has higher connectivity than the physical annealer does, then one incurs a penalty in the number of qubits needed to represent the Ising problem. For example, the largest fully connected Ising problem that can be represented in the D-Wave 2000-qubit quantum annealer is one that has $N=64$ spins $^{6}$; this limitation arises because the connectivity in this particular quantum annealer is very sparse (the connectivity graph has maximum degree six).

Superconducting circuits are one of the most prominent technologies for realizing quantum information processing devices, including quantum annealers, and they form the basis for many of the major projects to construct experimental quantum annealers $^{8-10}$. However, when qubit connectivity is achieved via physical pairwise couplers (as is the case for the efforts described in refs. ${ }^{8-10}$ ), there is a substantial engineering impediment to realizing full connectivity: each qubit would need $N-1$ physical couplers, and arranging such couplers spatially has proven to be impractical for large $N$. On the other hand, bus architectures have been demonstrated for superconducting-circuit qubits in the context of circuit-model quantum computing ${ }^{11-13}$, and busmediated interactions naturally provide all-to-all coupling ${ }^{11,14,15}$, with the use of one physical coupler per qubit. In this paper, we address the challenge of realizing full programmability of these all-to-all couplings, in the context of developing a quantum annealer.

In classical neuromorphic computing, a scheme providing full programmability has been proposed in an all-to-all-coupled system of Kuramoto oscillators using periodic modulation of each oscillator's phase ${ }^{16}$. Separately, it has long been known that two quantum oscillators can be coupled via phase modulation at their difference frequency ${ }^{17}$. In much more recent work, nonlinearoscillator-based qubits, whose operation relies on the continuousvariable nature of the oscillators, have been established as a promising building block for the realization of superconductingcircuit quantum annealers, owing partially to their resilience to photon loss ${ }^{18-20}$. We take inspiration from all of these lines of work, and show how we can combine them to design a quantum annealer that comprises a system of nonlinear-oscillator-based qubits having all-to-all coupling mediated by a bus, where periodic modulation of the oscillators is used to provide programmability in the couplings. Our main technical contribution is in showing how, through careful design of the modulation of each oscillator's instantaneous frequency, it is possible to enable full programmability, i.e., the realization of arbitrary connectivity between the qubits. We utilize the mathematical tools of Floquet theory ${ }^{21,22}$ to formulate and solve the design problem of engineering the desired interactions between oscillators, and we establish that the functionality of our dynamically coupled system is equivalent to that of a statically coupled system with pairwise physical couplers. We show that to gain arbitrary connectivity in our scheme, one needs to trade off the strength of the effective couplings, the main consequence of which is that longer coherence times are needed.

Our scheme applies generically to a variety of nonlinear oscillators, including those realized in platforms besides superconducting circuits, such as optics ${ }^{23-26}$ or nanomechanics ${ }^{27,28}$. However, for concreteness, we focus on Kerr parametric ${ }^{1}$ School of Applied and Engineering Physics, Cornell University, Ithaca, NY 14853, USA. ${ }^{2}$ E. L. Ginzton Laboratory, Stanford University, Stanford, CA 94305, USA. ${ }^{3}$ NTT Physics and
Informatics Laboratories, NTT Research, Inc., East Palo Alto, CA 94303, USA. ${ }^{4}$ These authors contributed equally: Tatsuhiro Onodera, Edwin Ng. ${ }^{\circledR}$ email: pmcmahon@ @cornell.edu 
oscillators $^{18,29,30}$ in which the Kerr nonlinearity is provided by a Josephson junction-i.e., Josephson parametric oscillators (JPOs) ${ }^{19,30-34}$. JPOs have been utilized in two other schemes for achieving programmable couplings: a proposed realization ${ }^{20}$ of the LHZ architecture ${ }^{35}$ (which requires $N(N-1) / 2$ physical qubits to represent $N$ spins), and an inductive-shunt scheme ${ }^{19}$ (which only provides $\mathcal{O}(N \log N$ ) programmable parameters, out of a total of $\mathcal{O}\left(N^{2}\right)$ in general).

In summary, the previously known approaches to building a quantum annealer with JPOs are, variously, incompatible with dense connectivity due to engineering limitations; requiring of a large overhead in the number of qubits and/or couplers (i.e., scaling with $\mathrm{N}^{2}$ ); or lacking full programmability. In contrast, our proposal uses a bus to provide all-to-all connectivity and a dynamical approach to coupling that enables full programmability of $\mathrm{N}$-spin Ising problems using a number of oscillators and couplers linear in $N$, and at the reasonable cost of coherence-time requirements that scale approximately linearly in $N$ for computationally interesting problem classes.

\section{RESULTS}

In this paper, we study the design of a quantum annealer whose purpose is to solve the Ising optimization problem, defined as finding the $N$-spin configuration $\sigma_{i} \in\{-1,+1\}(i=1, \ldots, N)$ that minimizes the classical spin energy $E(\sigma):=\sum_{j \neq i} C_{i j} \sigma_{i} \sigma_{j}$, where $C$ is a symmetric real matrix. A choice of $C$ specifies a problem instance to be solved, and $C$ can be interpreted as the adjacency matrix of a graph whose vertices are spins and whose edges represent spinspin interactions. In general, $C$ can have $\mathcal{O}\left(N^{2}\right)$ non-zero entries. It is desirable for a quantum annealer to be fully programmable, such that there are no restrictions on the structure of $C$, and that the annealer not use more than $N$ oscillators to represent a given $N$-spin problem, nor use more than $\propto N$ other physical components. In this paper, we show how this can be achieved using nonlinear oscillators in a bus architecture together with dynamically realized couplings designed via Floquet engineering.

Figure 1a shows an overview of our proposed architecture. The $N$ nonlinear oscillators of the quantum annealer are coupled to a common (bus) resonator. If the center frequencies of the oscillators are sufficiently far-detuned from the bus resonance, then the bus mediates a photon-exchange interaction between any pair of oscillators. In particular, denoting the annihilation operator for the ith oscillator as $\hat{a}_{i}$, the bus mediates interactions that contribute terms of the form $\hat{a}_{i}^{\dagger} \hat{a}_{j}+\hat{a}_{i} \hat{a}_{j}^{\dagger}$ to the system Hamiltonian ${ }^{11}$ which generically couples every oscillator to every other oscillator. Thus with $N$ nonlinear oscillators coupled to a bus, we can implement all-to-all coupling. However, the couplings up to this point are not programmable. The principal result in this paper is that we can engineer complete programmability of all the couplings by phase-modulating the oscillators in a specific way.

In our scheme, the oscillators are, to a good approximation, detuned from each other by multiples of a fundamental frequency $\Lambda$, such that the $k$ th-nearest neighbor of any given oscillator is detuned from it by $k \wedge$. Despite the presence of the bus, for $\Lambda$ sufficiently large the oscillators are effectively uncoupled in the absence of modulation (by the rotating-wave approximation). To effect dynamical coupling, each oscillator is controlled with a phase modulation (PM) signal containing harmonics of $\Lambda$, such that the resulting sidebands of each oscillator overlap in frequency with the center frequencies of the other oscillators. More precisely, the $i$ th oscillator is phase-modulated by $\delta \phi_{i}(t):=-\sum_{k=1}^{N-1} F_{i}^{(k)} \sin (k \wedge t)$, which causes its instantaneous frequency $\omega_{i}(t)$ to pick up a time-varying component $\dot{\delta} \phi_{i}(t)=-\sum_{k=1}^{N-1} F_{i}^{(k)} k \Lambda \cos (k \Lambda t)$. Here, the coefficients $F_{i}^{(k)}$ encode the strength of the $k$ th-harmonic component in the PM of Oscillator $i$, and they can be summarized by a matrix $F$ of dimension $N \times(N-1)$ whose $k$ th column consists of the elements $F_{1}^{(k)}, \ldots, F_{N}^{(k)}$. Intuitively, the strengths of the dynamically induced couplings are determined by the strengths of the sidebands, which in turn are controlled by the elements of $F$. Thus, the task of programming these couplings reduces to making an appropriate choice for $F$; we will present a method for choosing these coefficients shortly. Figure $1 \mathrm{~b}$ is a cartoon depicting the realization of this PM scheme for a representative 5-spin problem instance; the coefficients $F_{i}^{(k)}$ are shown on the left, while on the right are the power spectral densities (PSDs) of each oscillator's canonical position $x_{i}(t):=\cos \left(\int_{0}^{t} \omega_{i}\left(t^{\prime}\right) \mathrm{d} t^{\prime}\right)$. Each PSD shows a strong peak at its respective oscillator's center frequency, together with a

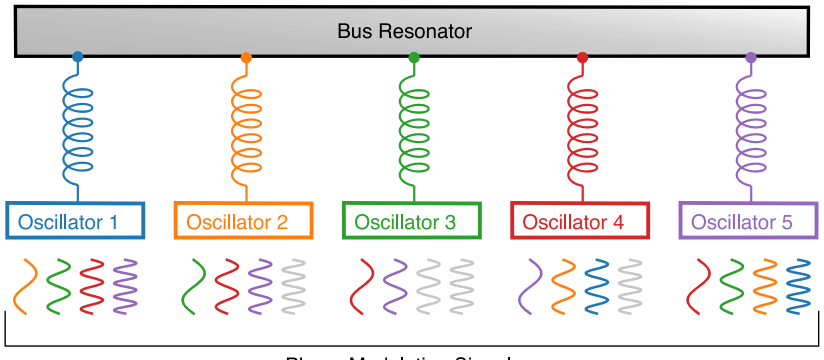

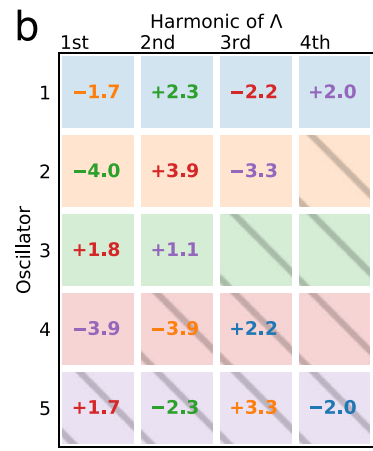

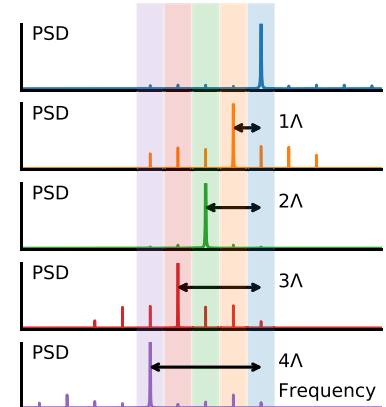

Fig. 1 Achieving fully programmable all-to-all coupling in oscillator networks via phase modulation. An implementation-independent overview of the architecture, showing an example with $N=5$ oscillators each coupled to a common bus resonator and each driven by a set of phase modulation (PM) signals. As depicted in (a), each oscillator is modulated by up to $N-1$ harmonics with frequencies $k \wedge$ for integers $k<$ $N$. The amplitude of each harmonic for each oscillator is given by an element in a phase-modulation matrix $F$, an example of which is shown on the left side of (b) (times a factor of 100 for clarity). The color of the PM signal on each oscillator indicates with which other oscillator the PM causes an interaction: for example, the $k=1$ harmonic PM applied to Oscillator 1 (shown in orange) causes it to couple with Oscillator 2, and the $k=2$ harmonic PM applied to Oscillator 1 (shown in green) causes it to couple with Oscillator 3. When a signal potentially mediates coupling to two other oscillators (e.g., $k=2$ for Oscillator 3), we color by the oscillator with the lower center frequency (larger oscillator index i); the signals colored gray are not applied as they would not create any useful couplings. As shown in the power spectral density (PSD) of the oscillators' canonical positions on the right side of $(\mathbf{b})$, the oscillators have center frequencies spaced by $\Lambda$; thus each PM signal at frequency $k \Lambda$ creates a primary sideband which overlaps in frequency with the center frequency of the neighbor $k$ oscillators away. Taken together, the effect of these sideband interactions is to generate a desired effective Ising-coupling matrix (see the Supplementary Information for the matrix used in this example). 
a

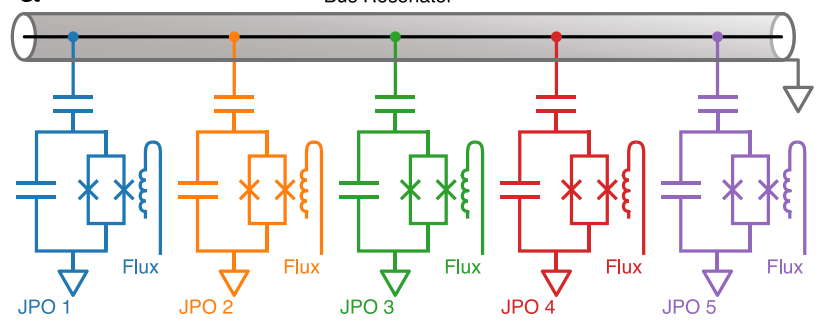

b

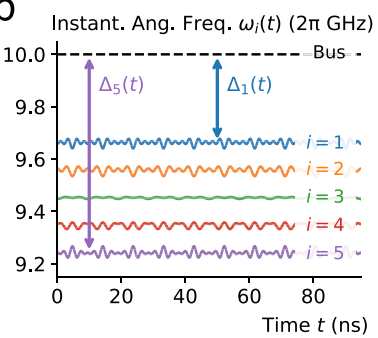

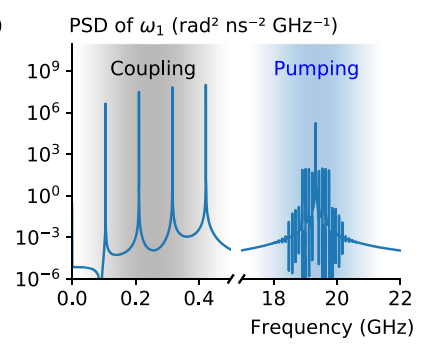

Fig. 2 A superconducting-circuits implementation of the dynamical-coupling architecture. a Josephson parametric oscillators (JPOs), whose frequencies can be modulated by a flux line, are coupled via a common microwave bus resonator. $\mathbf{b}$ Quantitative overview of the scheme for a JPO system, using the modulations shown in Fig. $1 \mathrm{~b}$ and depicting the instantaneous angular frequencies $\omega_{i}(t)$ of the JPOs, both in the time domain (left) and as a PSD of $\omega_{1}$ in the frequency domain (right). The PM signals manifest as modulations in the instantaneous frequency of each JPO, or, equivalently, in the detuning $\Delta_{i}(t)$ between the bus and Oscillator $i$. In the PSD, the PM signal appears in a lowfrequency band $(<0.5 \mathrm{GHz})$, while the parametric drive to the JPO (at twice the JPO's frequency) occurs as usual in a high-frequency band $\sim 20 \mathrm{GHz}{ }^{19,30}$. The parameters used for the calculation of numerical quantities in (b) are from the bus-coupled JPO model described in the Supplementary Information.

sidebands of various amplitudes (controlled by $F$ ) that overlap with the center frequencies of the other oscillators.

While our method is independent of the exact type of nonlinear oscillator used, we specialize our discussion in this paper to a superconducting-circuits realization. For concreteness, we consider JPOs ${ }^{19,30,31,33}$, although other superconducting-circuit-based oscillators are plausible choices as well ${ }^{36,37}$. In the absence of coupling, the eigenstates of the JPO are 0-phase and $\pi$-phase coherent states ${ }^{29,38}$; these two eigenstates can be used to encode, respectively, the spin-up and spin-down configurations of an Ising spin $^{18}$, and superpositions of these eigenstates (i.e., Schrödinger cat states) have been experimentally demonstrated ${ }^{33}$. Figure $2 a$ shows $N$ JPOs coupled to a superconducting resonator, which acts as the bus in this platform. Energy is supplied to each JPO by a flux line carrying a time-varying current. Conventionally, the dc component of the current determines the center frequency of the JPO, and an ac component at twice that frequency provides the parametric drive. In our scheme, the center frequency of each JPO is additionally modulated in time by $\dot{\delta} \phi(t)$, which is achieved by an additional modulation of the flux-line current corresponding to $\dot{\delta} \phi^{39,40}$. (In an alternative technological realization using a system of optical oscillators, this PM could potentially be applied by a physical phase modulator.)

For this JPO realization of a nonlinear-oscillator-based quantum annealer, Fig. $2 b$ shows a more quantitative picture of our dynamical-coupling scheme, with plots of the instantaneous angular frequencies $\omega_{i}(t)$ both in the time domain (for all the oscillators) and in the frequency domain (for the first oscillator only). First, on the time-domain plot, we see that the effect of the PM is to create oscillations in the instantaneous detuning $\Delta_{i}(t)$ between the fixed bus resonance frequency and $\omega_{i}(t)$; in the absence of these modulations (or on time-averaging), the oscillator frequencies are approximately evenly spaced. As expected, the PM consists of four harmonics; looking at the frequency-domain plot, we see that these harmonics lie in a "coupling band" with frequency content $\ll 1 \mathrm{GHz}$. These plots also illustrate two more technical features specific to our construction (see the Supplementary Information for more information). First, the deviations in oscillator frequency from the center (i.e., the modulation depths) are small compared with the mean values of the bus-oscillator detunings; this qualitative feature ensures that the native couplings $J_{i j}$ are approximately time-independent despite the PM. Second, there is a significant gap between the coupling band and the "pumping band" in which the parametric drive is realized; because of this separation of time scales, we can first design the flux-line currents to support the PM needed for dynamical coupling, while the parametric drive simply follows that modulation as needed. Since the ac part of the flux-line current is directly proportional to the ac part of $\omega_{i}(t)$ (see the Supplementary Information), we see that the entire control signal only requires $\sim 20 \mathrm{GHz}$ of bandwidth at most, which is realizable with current microwave technology.

We have thus far not explained how to choose the modulation coefficients $F$ for a given problem instance $C$. An intuitive, but incorrect, choice is to simply set $F_{i}^{(k)} \propto C_{i, i+k}$, since generating a sideband at frequency $k \Lambda$ on Oscillator $i$ intuitively causes it to interact with Oscillator $i+k$. However, this is not entirely accurate, as the symmetrically generated sideband at $-k \wedge$ (caused by the same $F_{i}^{(k)}$ coefficient) also leads to the same interaction with Oscillator $i-k$; for arbitrary $C$, these two interactions may need to differ in general. Furthermore, we must also consider higher-order sidebands as well: even if we were to only phase modulate Oscillator $i$ at frequency $k \Lambda$, weaker sidebands at $\pm 2 k \Lambda, \pm 3 k \Lambda$, and so on are also generated, causing interactions with Oscillators $i \pm$ $2 k, i \pm 3 k$, and so on, respectively. Thus, there is a nontrivial relation between $F$ and the desired couplings $C$, and $F$ needs to be chosen in a way such that the various contributions of $F$ to the effective couplings among oscillators combine appropriately to give the desired couplings $C$. To do this formally, we apply the mathematical tools of Floquet theory ${ }^{21,22}$.

First, to more explicitly define the problem we are trying to solve, a quantum annealer based on JPOs can be described by a (rotating-frame) Hamiltonian of the form ${ }^{18,30}$

$$
\hat{H}_{\text {static }}:=-\sum_{i}\left(\delta_{i} \hat{a}_{i}^{\dagger} \hat{a}_{i}+\frac{x}{2} \hat{a}_{i}^{\dagger 2} \hat{a}_{i}^{2}\right)+\sum_{i} \frac{r(t)}{2}\left(\hat{a}_{i}^{2}+\hat{a}_{i}^{\dagger 2}\right)-\lambda_{C} \sum_{j \neq i} C_{i j} \hat{a}_{i}^{\dagger} \hat{a}_{j} \text {, }
$$

where $X$ is the Kerr nonlinear rate, $\delta_{i}$ is the detuning between Oscillator $i$ and the half-harmonic of its parametric drive, and $r(t)$ is the (slowly time-varying) amplitude of the parametric drives. In this work, we choose the detuning to be $\delta_{i}=\lambda_{c} \sum_{j=1}^{N}\left|C_{i j}\right|$ as prescribed by Ref. ${ }^{18}$, and the parametric drive to be a clamped linear $\operatorname{ramp} r(t)=r_{\max } \min \left(t / T_{\text {ramp }}, 1\right)$. Here, $\lambda_{c}$ is a problemstrength parameter dictating the strength of the oscillatoroscillator couplings $C_{i j}$ (which are normalized in this work to $\left.\max _{j \neq i}\left|C_{i j}\right|=1\right)$. One way to realize such a Hamiltonian is to have $\mathcal{O}\left(N^{2}\right)$ physical pairwise couplers, which can be programmed given a desired $C_{i j}$; these programmed couplings can then be held static throughout the annealing process while the parametric drive is varied.

By contrast, our goal is to realize this annealing Hamiltonian via dynamical control of the effective couplings among the oscillators. As a result, we start instead with the (rotating-frame) Hamiltonian 
(see the Supplementary Information for a derivation)

$$
\begin{aligned}
\hat{H}_{\text {dynamical }}:= & -\sum_{i}\left(\delta_{i} \hat{a}_{i}^{\dagger} \hat{a}_{i}+\frac{x}{2} \hat{a}_{i}^{\dagger 2} \hat{a}_{i}^{2}\right)+\sum_{i} \frac{r(t)}{2}\left(\hat{a}_{i}^{2}+\hat{a}_{i}^{\dagger 2}\right) \\
& -\sum_{j \neq i} J_{i j} \exp \left[-\mathrm{i}\left(\Lambda(i-j) t+\delta \phi_{j}(t)-\delta \phi_{i}(t)\right)\right] \hat{a}_{i}^{\dagger} \hat{a}_{j},
\end{aligned}
$$

which is the same as $\hat{H}_{\text {static }}$ with the exception of the final coupling term. In this case, $J_{i j}$ are the bus-mediated coupling rates natively present in the system but which are not fully programmable in general (see the Supplementary Information for the exact form). By detuning the oscillators relative to one another by multiples of $\Lambda$ and applying PM to the oscillators according to $\delta \phi_{i}(t)$, the result is the "native" coupling term $\hat{H}_{\text {native }}:=-\sum_{j \neq i} J_{i j} \exp [-\mathrm{i}(\Lambda(i-j) t+$ $\left.\left.\delta \phi_{j}(t)-\delta \phi_{i}(t)\right)\right] \hat{a}_{i}^{\dagger} \hat{a}_{j}$. The time-varying phase factor is due to the $\mathrm{PM}$ of the $\Lambda$-detuned oscillators, and control over its time dependence forms the core of our dynamical-coupling scheme.

If we denote the coupling term in the static annealing Hamiltonian (1) as $\hat{H}_{\text {target }}:=-\lambda_{C} \sum_{j \neq i} C_{i j} \hat{a}_{i}^{\dagger} \hat{a}_{j}$, then the goal is to achieve $\hat{H}_{\text {native }} \approx \hat{H}_{\text {target, }}$ under some appropriate sense of the approximation. As previously mentioned, we choose the modulations to be $-\sum_{k=1}^{N-1} F_{i}^{(k)} \sin (k \wedge t)$, which means that $\hat{H}_{\text {native }}$ is periodic with frequency $\Lambda$. If $\Lambda$ is much larger than all other system timescales, then the results of Floquet theory allow us to make the Floquet approximation $\hat{H}_{\text {native }} \approx-\lambda_{c} \sum_{j \neq i} C_{\text {eff } i j} \hat{a}_{i}^{\dagger} \hat{a}_{j}$ where

$$
C_{\text {effij }}:=\int_{0}^{2 \pi / \Lambda} \frac{J_{i j}}{\lambda_{C}} \cos \left[(i-j) \Lambda t+\sum_{k=1}^{N-1}\left(F_{i}^{(k)}-F_{j}^{(k)}\right) \sin (k \Lambda t)\right] \frac{\mathrm{d} t}{2 \pi / \Lambda}
$$

describes the effective couplings between oscillators $i$ and $j$ due to all the sideband interactions. (More formally, this approximation is the leading-order term in the Floquet-Magnus expansion of $\hat{H}_{\text {native }}$ in $1 / \Lambda$.) Thus, all that remains is to choose the coefficients in $F$ such that $C_{\text {eff } i j} \approx C_{i j}$. As discussed in the Supplementary Information, while it is possible to solve for $F$ by direct numerical nonlinear optimization, there are a number of ways to make this precomputation step more tractable and robust. In particular, one can consider a second-order Taylor expansion of $C_{\text {eff } i j}$ (intuitively, by considering effective interactions only up to the second-order sidebands) and obtain a system of quadratic equations that can be numerically solved.

To demonstrate the effectiveness of our approach, we perform numerical simulations of both the statically coupled system governed by $\hat{H}_{\text {static }}$ as well as the dynamically coupled system governed by $\hat{H}_{\text {dynamical }}$ (with an appropriate choice of $F$ given $C$ ), and we show that they achieve nearly indistinguishable results, as one would expect if one has achieved $\hat{H}_{\text {dynamical }} \approx \hat{H}_{\text {static }}$. Figure $3 a$ shows the results of simulating the quantum evolution of both systems as they perform quantum annealing on a two-spin problem, where the coupling between the spins is antiferromagnetic (Table 1). The joint quantum states of the dynamically coupled oscillators show that the system is initially in a vacuum state (with equal probability on each of the four possible spin configurations); by the end of the evolution, the system is in a superposition $\frac{1}{\sqrt{2}}(|\uparrow \downarrow\rangle+|\downarrow \uparrow\rangle)$ in the qubit basis, and would produce one of the correct ground-state spin configurations $\uparrow \downarrow$ or $\downarrow \uparrow$ upon measurement. We see that the evolution of the success probability to obtain a ground state is nearly identical for both architectures at all times, suggesting that the dynamically coupled system is closely mimicking the behavior of the statically coupled system, as desired.

Figure $3 b$ shows the same evolution of the success probability for a particular $N=4$ problem instance, chosen from the class of finite-range, integer-valued Sherrington-Kirkpatrick (SK) instances studied in a foundational quantum-annealing benchmark work ${ }^{41}$; in particular, we utilize range-7 graphs (SK7) (see the Supplementary Information for details about instance generation). We again see that the evolutions of the success probabilities match very closely. Aside from the success probability, Fig. $3 c$ shows the projections of the quantum state onto the spin configurations (of which there are 16 for $N=4$ ), which demonstrates that the two architectures produce nearly indistinguishable evolutions in the projections as well.

In Fig. 3d, we show the results from $N=4$ simulations for 100 different randomly generated problem instances (again drawn from the SK7 problem class). We simulate both the statically coupled and dynamically coupled quantum annealers and show the correlations between their respective success probabilities. To explore the effects of decoherence due to photon loss, we also examine these correlations for three different values of cavityphoton decay rate $k$ (which is related to the cavity-photon lifetime as $\left.T_{\text {cav }}:=1 / \kappa\right)$. We see that even in the cases of non-zero loss $(\kappa>$ $0)$, both annealers are still able to find ground states with high success probability, which demonstrates the loss-resilience results found in previous work ${ }^{19,20}$. Just as importantly, the correlations remain strong in the presence of photon losses. As a result, the loss resilience of the statically coupled architecture carries over to the dynamically coupled architecture, and even for those instances where the statically coupled system suffers in success probability due to photon loss events, the dynamically coupled system follows its behavior as expected.

While it would be desirable to validate our theoretical results with quantum simulations having more than $N=4$ spins, full quantum simulations are prohibitively expensive for $N>4$. Fortunately, for an oscillator-based quantum-optical system, there is a natural set of classical equations of motion (EOMs) which one can derive from the quantum model; formally, this consists of replacing the annihilation operators $\hat{a}_{i}$ with coherent-state amplitudes $a_{i} \in \mathbb{C}$ in the quantum Heisenberg EOMs. Such a set of classical EOMs does not fully capture the dynamics of the system in the quantum regime, but we can nevertheless simulate these classical EOMs for both the statically coupled and dynamically coupled annealers and compare their dynamical behavior. If the two systems correspond well, we gain further confidence that our theoretical results can lead to the desired performance on a dynamically coupled quantum annealer. Figure 4 shows the results of simulating these classical EOMs for both architectures on SK7 problems. Figure 4a shows the evolutions of the success probabilities for a particular $N=10$ problem instance, while Fig. $4 \mathrm{~b}$ shows the evolutions of the field amplitudes $a_{i}(t)$. We see that both the success probabilities and the oscillator amplitudes of the two architectures match well. Figure $4 \mathrm{c}$ shows the distribution of success probabilities for different problem sizes $N$ up to $N=50$, using 100 problem instances for $N \leq 30$, and 30 instances for $N=50$; the corresponding correlation plots for $N=8$ and $N=30$ are also shown to the bottom of the figure (additional correlation plots can be found in the Supplementary Information). There is good agreement between the simulation results of the statically and dynamically coupled systems: the success probability as a function of $N$ scales in the same way, and the correlation plots show similar performance on an instance-byinstance basis.

Having shown that the dynamically coupled system indeed reproduces the behavior of the desired quantum annealer, we now discuss an important tradeoff inherent in our PM scheme for dynamical coupling. Examining the expression (3) for $C_{\text {eff, }}$ we see that a key parameter is the ratio between the native couplings $J_{i j}$ and the desired problem-strength parameter $\lambda_{\mathrm{C}}$. For simplicity, we design the bus-resonator interactions in such a way that the native couplings are approximately constant (see the Supplementary Information for more details). Thus, let us assume that $J_{i j} \approx \lambda_{\mathrm{J}}$, where $\lambda_{J}>0$ characterizes the rate at which photons are exchanged via the bus (i.e., the strength of the native couplings). 
a

a
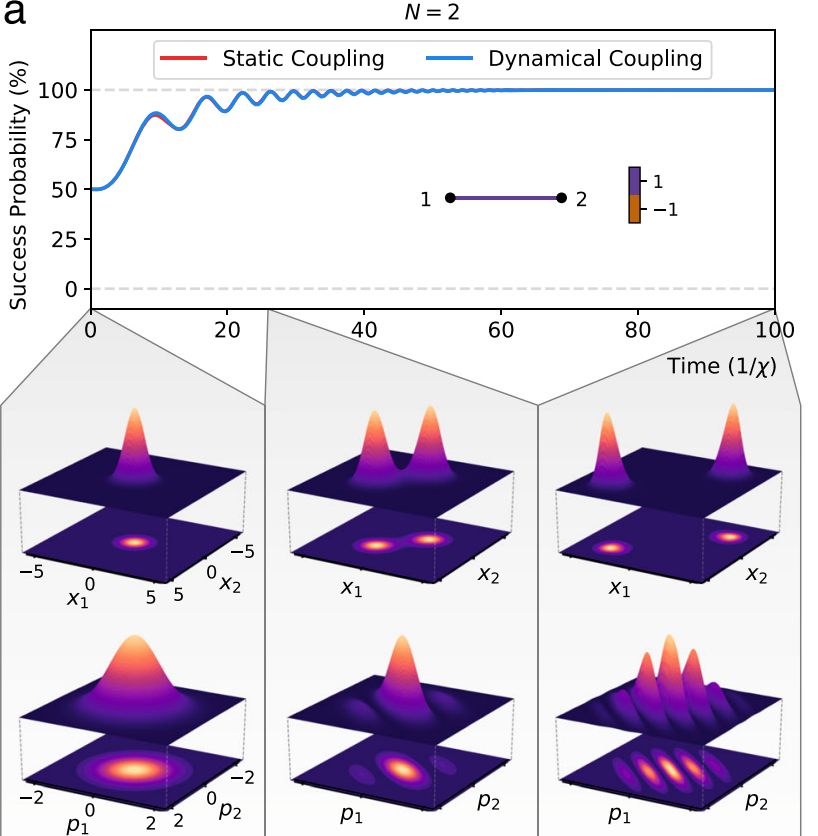

b
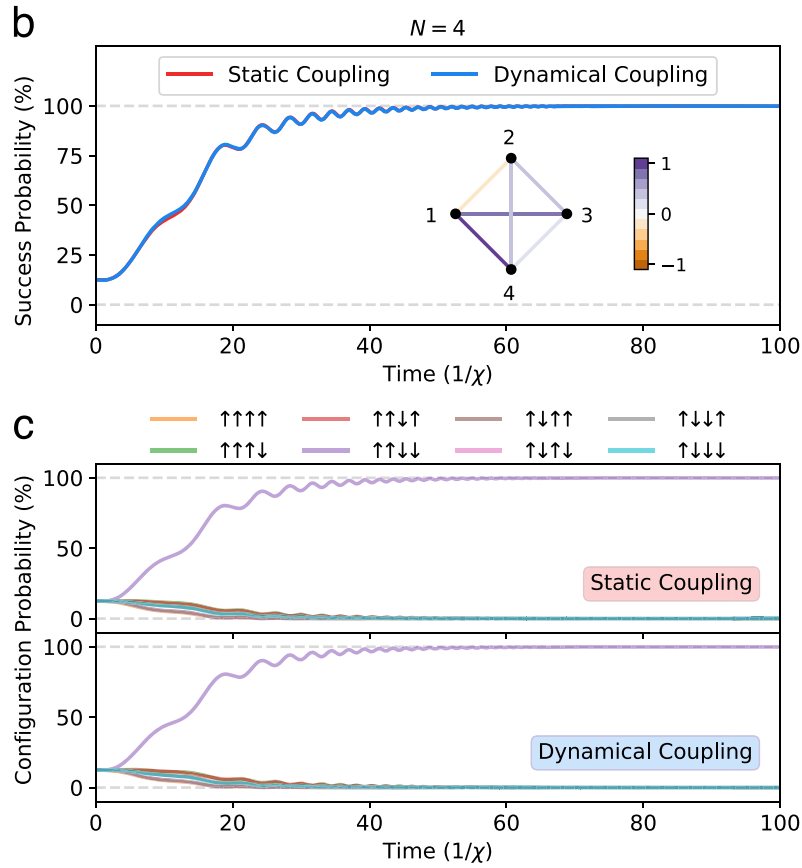

d
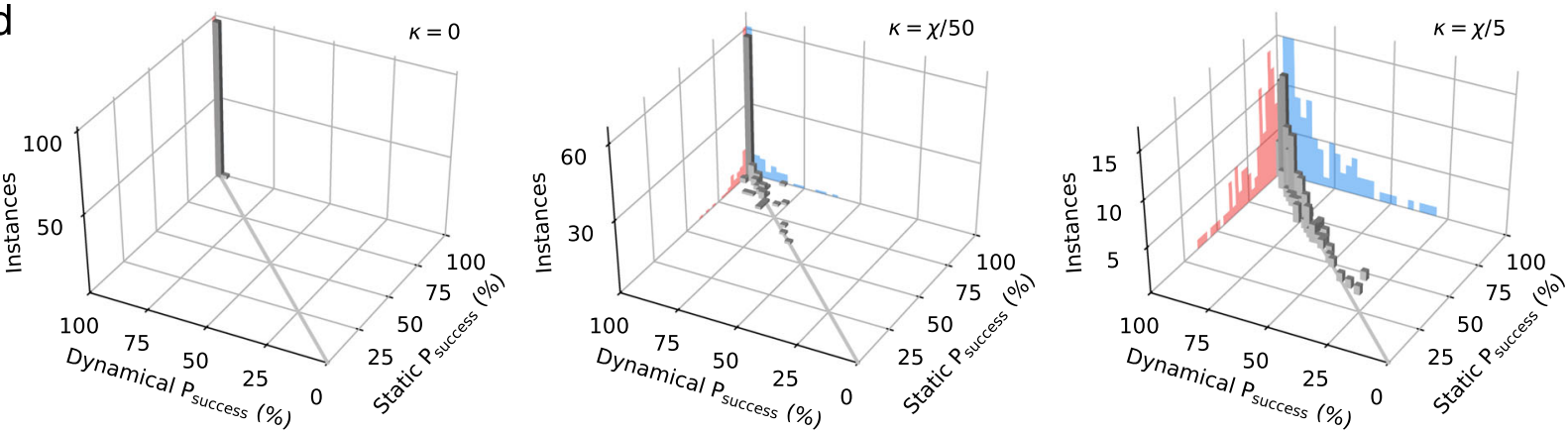

Fig. 3 Quantum simulations. a, b Evolution of the success probability of finding a ground state of the inset Ising problem instances, for both a system whose couplings are implemented by static pairwise physical couplers (static coupling) and a system using this work's dynamicalcoupling approach (dynamical coupling), in the case of no dissipation. Shown to the bottom of (a) are the joint quantum states of the oscillators (represented as probability distributions $\left|\psi\left(x_{1}, x_{2}\right)\right|^{2}$ and $\left|\psi\left(p_{1}, p_{2}\right)\right|^{2}$ in canonical position and momentum coordinates, respectively) at three different times for the dynamically coupled system. c The probabilities of obtaining each of the possible spin configurations (i.e., configuration probability) as a function of the evolution time, for the problem instance and simulations shown in (b). Since the problem does not have any external fields, each spin configuration shown is degenerate to flipping every spin; for brevity, we have added together the probabilities corresponding to these degenerate configurations. d Correlation matrices of success probabilities $P_{\text {success }}$ (evaluated at the end of the computation) between the statically coupled and dynamically coupled systems, for 100 instances of the SK7 problem class. (For the trajectory simulation data used to generate these correlation matrices, see the Supplementary Information.) Here, we also introduce a nonzero cavity-photon decay rate $\kappa$, to show that the correlations are high even in the presence of photon losses. Projected to the sides in blue and red are the corresponding histograms for the dynamically coupled and statically coupled systems, respectively. For simulation parameters and methodology, see Table 1 and "Methods".

Table 1. System parameters of the statically and dynamically coupled annealers for representative quantum (left) and classical (right) simulations in this work.

\begin{tabular}{|c|c|c|c|c|c|c|c|c|c|c|c|}
\hline $\begin{array}{l}\text { Quantum } \\
\left(N_{\text {Fock }}=12\right)\end{array}$ & $r_{\max } / X$ & $T_{\text {ramp } X}$ & $\lambda_{C} / x$ & $\lambda_{\jmath} / X$ & $1 / x$ & Classical & $r_{\max } / X$ & $T_{\text {rampX }}$ & $\lambda_{C} / X$ & $\lambda_{\jmath} / X$ & $1 / x$ \\
\hline$N=2, k / X=0$ & 5.0 & 100 & 2.0 & 10 & 500 & $N=8$ & 5.0 & 100 & 0.50 & 5 & 1000 \\
\hline$N=4, k / X=2 \times 10^{-2}$ & 4.5 & 25 & 1.2 & 6 & 500 & $N=20$ & 5.0 & 100 & 0.20 & 5 & 1000 \\
\hline$N=4, k / X=2 \times 10^{-1}$ & 4.0 & 5 & 1.4 & 7 & 500 & $N=30$ & 5.0 & 100 & 0.13 & 5 & 1000 \\
\hline
\end{tabular}

Here, $N_{\text {Fock }}$ denotes the Fock space dimension allocated for each JPO mode in the quantum simulations; for reference, the expected photon number in each JPO mode at $t \geq T_{\text {ramp }}$ is approximately $r_{\max } / X$. 

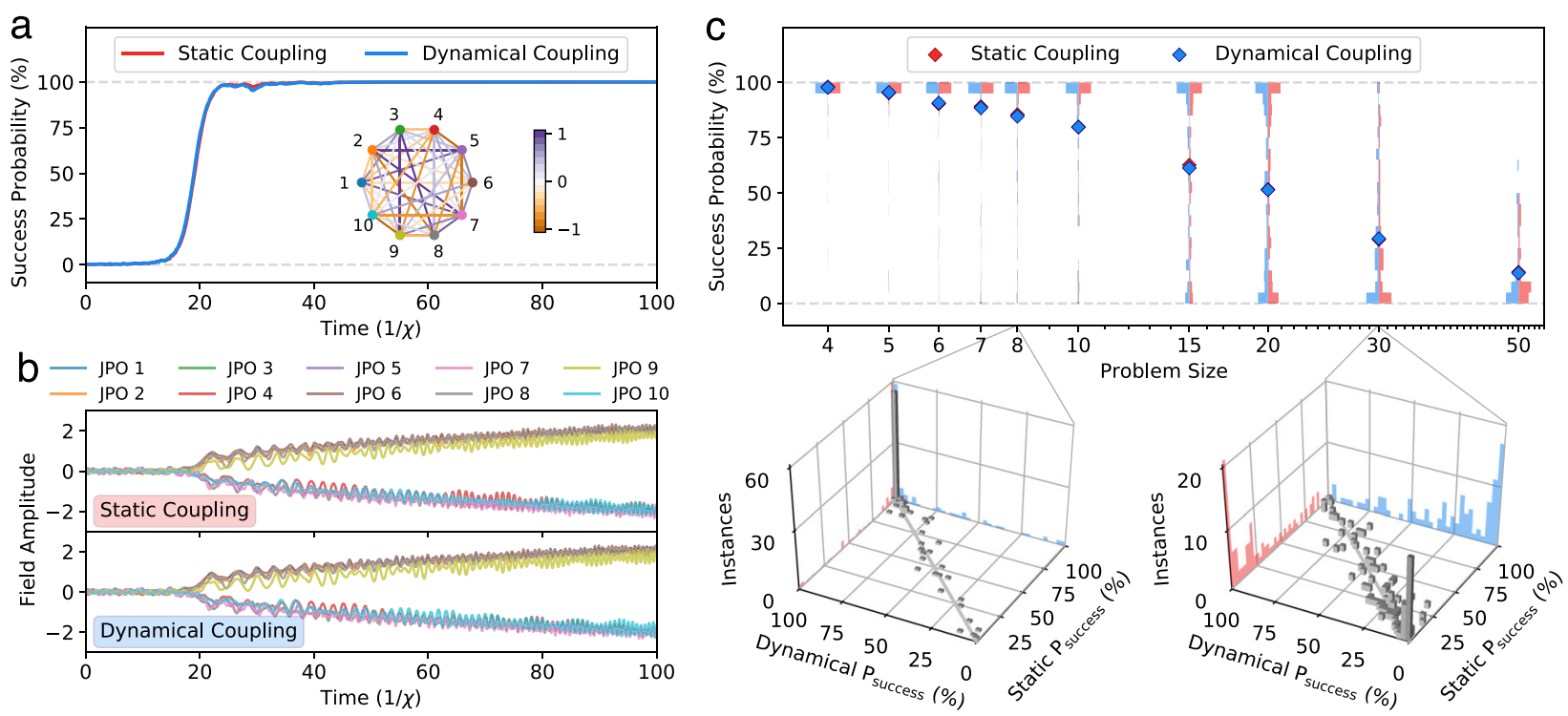

Fig. 4 Simulations of classical equations of motion. a Evolution of the success probability of finding a ground state of the inset 10-spin Ising problem instance, for both a system whose couplings are implemented by static pairwise physical couplers (static coupling) and a system using this work's dynamical-coupling approach (dynamical coupling). To estimate the probability, we sample random initial conditions according to a Gaussian distribution (see the Supplementary Information, ref. ${ }^{18}$ ). $\mathbf{b}$ Trajectories of the classical field amplitudes of each of the 10 oscillators, for one of the samples constituting the simulation in (a). c Histograms of the success probability $P_{\text {success }}$ for various problem sizes $N$, each estimated using 100 instances of the SK7 problem class. The diamonds indicate the average success probability, while the horizontal bars show the histogram. For $N=8$ and $N=30$, the correlation matrices between statically and dynamically coupled systems are shown at the bottom (For the correlation matrices for all other problem sizes, see the Supplementary Information). Projected to the sides of the correlation matrices, in blue and red, are the corresponding histograms for the dynamically coupled and statically coupled systems, respectively. For simulation parameters and methodology, see Table 1 and "Methods".

Under this framework, we can identify a "dynamical-coupling parameter" $\eta:=\lambda_{C} / \lambda_{\jmath}$, which, intuitively, captures the "cost" of the dynamical-coupling scheme as it sets the scale for how much effective coupling we obtain for a given amount of native coupling provided to the scheme. As we discuss later, the absolute scale of $\lambda_{J}$ is generally hardware-limited, while for any given problem, there is a fixed value of $\lambda_{C} / X$ required to ensure successful annealing (see the Supplementary Information for details). Given these constraints, larger values of $\eta$ allow for larger operating values for $X$, which is advantageous in the presence of a fixed cavity-photon lifetime. (A full account of the hierarchy of timescales and the chain of parameter requirements is given in the Supplementary Information.) Therefore, it is desirable to use as high a value of $\eta$ as possible.

On the other hand, a clear requirement for dynamical coupling to work well is the ability to obtain $C_{\text {eff }} \approx C$ (so that $\hat{H}_{\text {dynamical }} \approx \hat{H}_{\text {static }}$ ) for any desired $C$. When $\eta$ becomes large, however, the prefactor $J_{i j} / \lambda_{C} \approx 1 / \eta$ in (3) becomes small. As discussed in the Supplementary Information, this effect can become detrimental to our ability to obtain $C_{\text {eff }} \approx C$ : intuitively, the maximum magnitude of the elements of $C$ is by construction unity, but for a sufficiently small prefactor for the integral (i.e., sufficiently small $1 / \eta$ ), it becomes impossible to find a suitable set of coefficients $F_{i}^{(k)}$ that could allow the integral over the cosine to compensate. This phenomenon is demonstrated in Fig. 5. Given a particular target coupling matrix $C$, Fig. 5a shows, over a range of values for $\eta$, the $C_{\text {eff }}$ matrices corresponding to the optimal $F$ matrix found by our (second-order-Taylor-expansion-based) numerical routine (see the Supplementary Information). We see that when $\eta$ is chosen to be too large, the correspondence between $C$ and $C_{\text {eff }}$ is rather poor. However, by decreasing $\eta$, one can achieve improved accuracy. More quantitatively, Fig. $5 \mathrm{~b}$ shows the maximum element-wise error $\left(\max _{i \neq j}\left|C_{\text {eff } i j}-C_{i j}\right|\right)$, as a function of $\eta$ and problem size $N$ (averaged over an ensemble of 100 instances for each $N$ ). To show how the resulting error depends on the structure of $C$, we consider three problem classes: the SK7 class discussed above, the class of unweighted MAX-CUT problems with $50 \%$ edge density, and the class of unweighted MAX-CUT problems on cubic graphs (see the Supplementary Information for details about instance generation). As expected from our intuition about the role of $\eta$, the particular scaling of the error with $\eta$ depends on the type of problems considered: for SK7, the required $\eta$ goes approximately as $\eta \propto 1 / N$, while for dense MAX-CUT, $\eta \propto 1 /(N \log N)$; in contrast with these two dense problem classes, cubic MAX-CUT problems require $\eta \propto 1 / \log N$. From this figure, we see that, assuming we can tolerate an upper limit for the error in $C_{\text {eff }}$ (say, of 3\%), there is a maximum value for $\eta$ that we are able to use for any given $N$. By staying below this maximal value, we can ensure feasible solutions for the modulation coefficients $F_{i}^{(k)}$ which generate effective couplings to within the acceptable error. In the Supplementary Information, we derive for each problem class an explicit, functional form of $\eta$ with respect to problem size $N$ such that we are guaranteed an error of at most $3 \%$, but which is also not so conservative that the resulting nonlinear rate $X$ is unnecessarily low.

Finally, to study the technological feasibility of our dynamicalcoupling scheme, it is important to understand how this tradeoff between the accuracy of the effective couplings and the nonlinear rate-in combination with the requirements for the Floquet approximation - translate into concrete scaling requirements for hardware figures of merit as a function of problem size. As previously mentioned, two relevant hardware limitations are the maximum coupling rate $g_{\max }$ between each oscillator and the bus, as well as the maximum realizable detuning $\Delta_{\max }$ between the bus and each oscillator. In the presence of these two limitations, we find (see the Supplementary Information for a full analysis) that there is a maximum allowable nonlinear rate $X_{\max }$ for dynamical coupling to work.

In Fig. 6a, we show $X_{\max }$ as a function of problem size $N$, upon varying the hardware limits $g_{\max }$ and $\Delta_{\max }$ (for the SK7 problem 

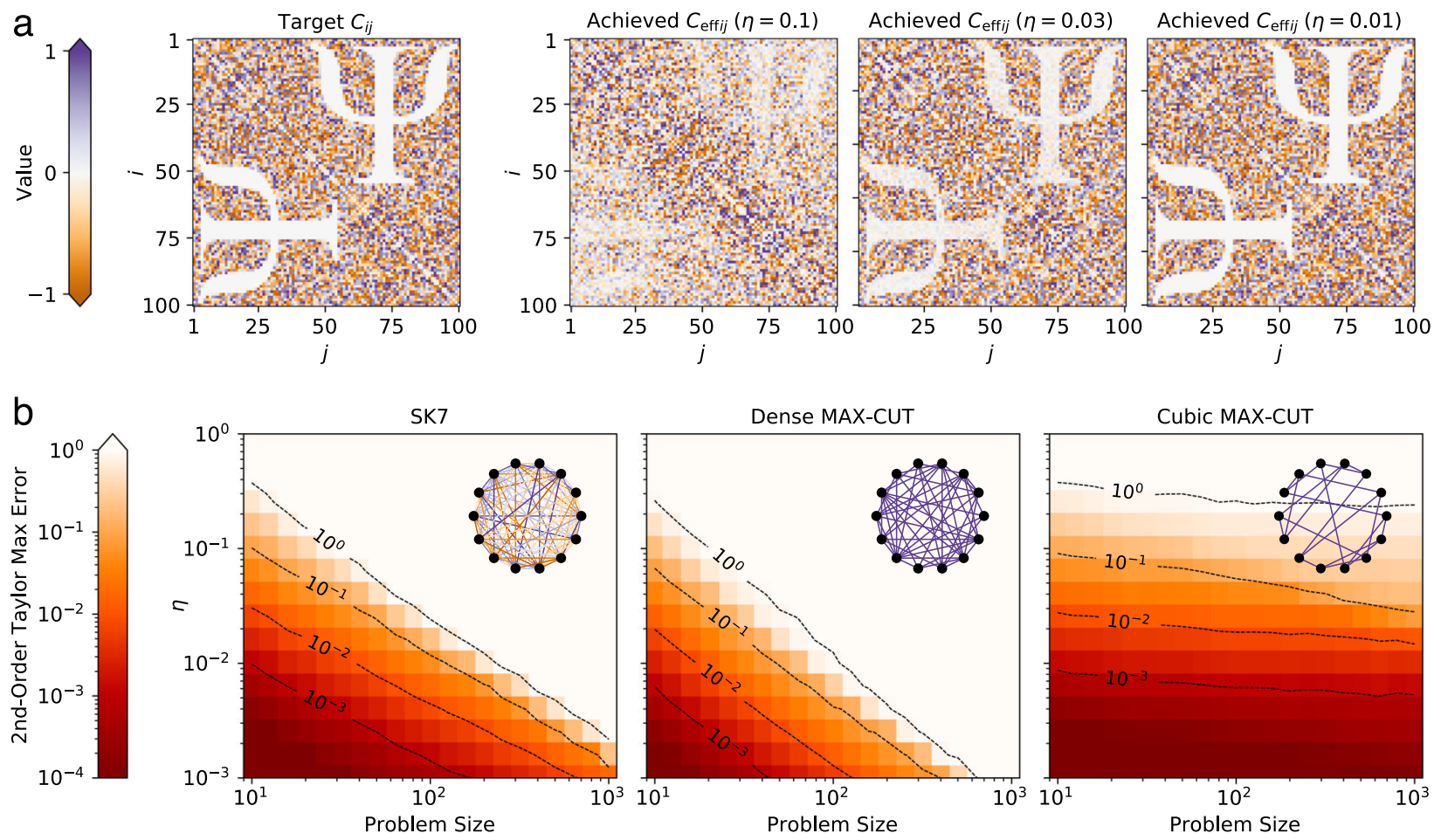

Fig. 5 Accuracy of the effective couplings. a Comparison of the effective coupling matrices $C_{\text {eff }}$ (target Ising problem matrix $C$ shown to the left) for different settings of the dynamical-coupling parameter $\eta$, which sets the ratio between the effective coupling strength and the strength of the native coupling provided via the bus. As $\eta$ is decreased, the accuracy of the $C_{\text {eff }}$ increases. $\mathbf{b}$ The maximum entry-wise error in the effective coupling matrix $C_{\text {eff, }}$ averaged over 100 problem instances for each size $N$, as a function of the dynamical-coupling parameter $\eta$. We show the scaling for three different classes of Ising problems, with illustrative examples of instances shown as insets. For all panels, the modulation matrix $F$ used to produce $C_{\text {eff }}$ is found by a numerical optimizer that attempts to maximize the accuracy by using a second-order Taylor approximation to (3), with native couplings $J_{i j}$ constructed to be approximately uniform (see the Supplementary Information).

class; see the Supplementary Information for MAX-CUT). We observe that at fixed values of $g_{\max }$ and $\Delta_{\max }, X_{\max }$ decreases as $N$ increases. To more concretely interpret the implications of the requirement $X \leq X_{\max }$, we note that $k \ll X$ is a necessary condition for the annealer to operate in the quantum, rather than the dissipative, regime. Thus, the requirement of $x \leq X_{\max }$ translates directly to a minimum required value for the cavity-photon lifetime $T_{\text {cav }}=1 / \kappa$. Taking $\kappa=X / 5$ for concreteness, we plot the minimum required cavity-photon lifetime $T_{\text {cav }}$ on the right axis of Fig. $6 \mathrm{~b}$ as a function of $N$, for $g_{\max } / 2 \pi=20 \mathrm{MHz}$ and $\Delta_{\max } / 2 \pi=5 \mathrm{GHz}$. This plot indicates that $T_{\text {cav }} \approx 100 \mu \mathrm{s}$ is required to achieve $N=1000$.

The values for $g_{\max }$ and $\Delta_{\max }$ are readily achievable experimentally; regarding the $T_{\text {cav }}$ requirement, transmon qubits with $T_{1}$ times of around $50 \mu \mathrm{s}-100 \mu \mathrm{s}$ have been measured ${ }^{42,43}$. We also mention that, in the spirit of refs. ${ }^{3,8}$, one could also ignore this latter requirement on $T_{\text {cav }}$ and build a system with the best $T_{\text {cav }}$ one can achieve-even if it is smaller than the required $T_{\text {cav }}$ to satisfy $k \leq X / 5$-and experimentally explore the performance of a highly dissipative quantum annealer. For a small experimental demonstration that still obeys the $\kappa \leq X / 5$ requirement, $T_{\text {cav }} \sim 10 \mu \mathrm{s}$ should be sufficient to realize an $N=10$ version of the system.

\section{DISCUSSION}

There are two fundamental tradeoffs that one is making by adopting our dynamical-coupling architecture versus a staticcoupling architecture: firstly, it is necessary to perform some classical precomputation to obtain the PM coefficients (i.e., the matrix $F$ ), and secondly, as alluded to in the discussion about the dynamical-coupling parameter $\eta$, each oscillator's cavity-photon lifetime in a dynamical-coupling architecture will need to be longer than it would in a static-coupling architecture. In exchange for accepting these two downsides, one is able to build a fully programmable, fully connected $\mathrm{N}$-spin quantum annealer with a number of qubits and couplers that only scales as $N$, as opposed to current proposals for statically coupled quantum annealers, which require a number of qubits and/or couplers that scales as $N^{2}$.

Our method for computing $F$-by minimizing a second-order Taylor approximation of effective coupling error-requires only $\mathcal{O}\left(N^{3}\right)$ time, which is efficient, and remarkably so given that the Ising problem matrix contains $\mathcal{O}\left(N^{2}\right)$ entries in general, hence the runtime could at best be $\mathcal{O}\left(N^{2}\right)$. Moreover, in our numerical experiments, the wall-clock times when running our implementation of the method on a single-core processor were $\sim 2$ min for $N=1000$, so the precomputation time should not be a significant practical concern, given the difficulty of solving hard instances of Ising problems.

With regards to the required cavity-photon lifetime, we have provided (in the Supplementary Information) a prescription for the parameters in the dynamical-coupling scheme such that the error in the realized couplings will be at most $\sim 3 \%$, and we have computed the resulting cavity-photon-lifetime requirement as a function of $N$. Current experimental efforts with $\mathrm{JPOS}^{33,34}$ have shown promising development. In particular, a single cat qubit with a JPO was demonstrated in Ref. ${ }^{34}$, and the qubit had $T_{1}$ and $T_{2}$ values of $15.5 \mu \mathrm{s}$ and $3.4 \mu \mathrm{s}$ respectively. While the use of dynamic flux modulation to induce multimode couplings among JPOs (with or without a bus) has not yet been experimentally investigated to the best of our knowledge, flux modulation has been used to construct two-qubit gates between transmon qubits $^{40}$ as well as to couple multiple modes of a transmission line ${ }^{44}$. Meanwhile, bus-mediated multi-qubit couplings achieving all-to-all entanglement have now reached the scale of up to 20 

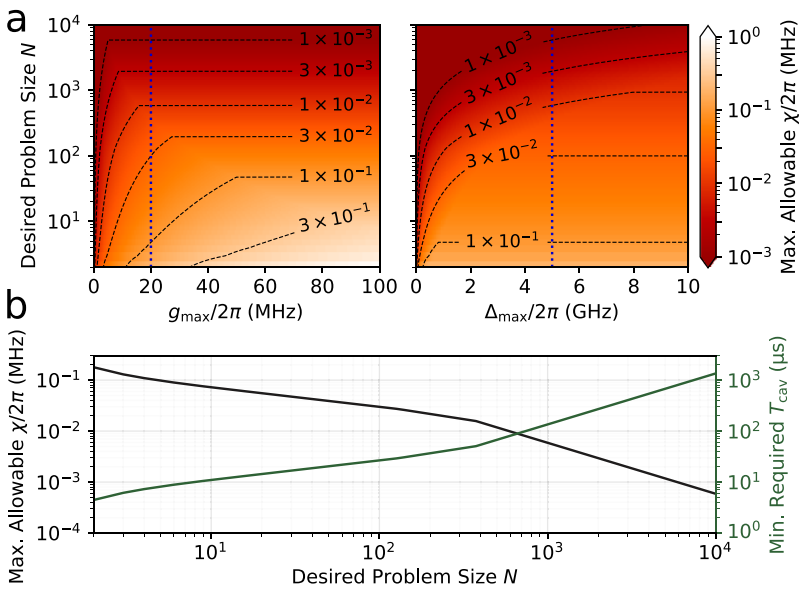

Fig. 6 Scaling requirements for SK7 problems. Due to the tradeoff between accuracy of the effective couplings and the required strength of native couplings, hardware limitations on the achievable bus-oscillator detunings and bus-oscillator couplings impose a maximum allowable nonlinear rate $X$ in the system. a The maximum allowable nonlinear rate as a function of the desired problem size $N$ and the hardware-limited bus-oscillator coupling rate $g_{\max }$ (left, with $\Delta_{\max } / 2 \pi=5 \mathrm{GHz}$ ) and the hardware-limited bus-oscillator detuning $\Delta_{\max }$ (right, with $g_{\max } / 2 \pi=20 \mathrm{MHz}$ ). Dashed vertical lines in blue indicate the slice of these plots shown in (b) below. $\mathbf{b}$ The black line (left axis) shows the maximum allowable nonlinear rate as a function of problem size for fixed $\Delta_{\max } / 2 \pi=5 \mathrm{GHz}$ and $g_{\max } / 2 \pi=20 \mathrm{MHz}$. The green line (right axis) shows the consequence of the maximum allowable nonlinear rate for the minimum required cavity-photon lifetime $T_{\text {cav, }}$ assuming that the quantum annealer requires $X \geq 5 \mathrm{~K}$. See the Supplementary Information for the corresponding scaling requirements for MAX-CUT problem classes.

transmon qubits on a single chip ${ }^{15}$. These experimental milestones, together with the generally rapid progress in superconducting circuit hardware, indicate that the coherence-time requirements found in our scaling analysis $-10 \mu \mathrm{s}$ for a small-scale near-term demonstration and $100 \mu$ s for a 1000-spin machineare within optimistic experimental reach.

In the current approach from D-Wave Systems ${ }^{6,8,45}$, as well as the proposed approaches in refs. 20,35 , realizing a 1000-spin quantum annealer for fully connected problems would require on the order of $10^{6}$ qubits. This stands in contrast to our proposed architecture, which would require only 1000 oscillators to achieve the same number of spins. Beyond the engineering expense of implementing a relatively larger number of qubits, quantum annealers using problem embedding can also suffer from an additional exponential degradation in success probability ${ }^{6}$. The dynamical-coupling approach uses the minimal number of physical qubits possible, and hence avoids this additional penalty in performance.

Outside the context of quantum annealing for solving optimization problems, our work has a strong connection with Floquet engineering of quantum simulators and quantum spin chains $^{46-50}$. A quantum annealer, in addition to being an optimization machine, is also a realization of a quantum simulator of the transverse-field Ising model ${ }^{51}$. We anticipate that our techniques for dynamic control of the couplings in a spin system will also enable the realization of novel simulation capabilities for more general spin Hamiltonians. Furthermore, our technique and derivations are not restricted to simulators of spin systems, but apply directly to generic bosonic simulators, and may allow more complex Hamiltonians to be engineered than are currently realized with pairwise physical couplers in Bose-Hubbard simulators built with superconducting circuits ${ }^{52-56}$.

\section{METHODS}

Ising problem classes and problem instance generation We consider problem instances drawn from the following three specific classes of Ising problems, characterized by the statistical distribution of the $C_{i j}$ couplings specifying the Ising problem:

- Integer Sherrington-Kirkpatrick graphs with range 7 (SK7) ${ }^{41}$ : Every upper-triangular element of $C$ is independently chosen with equal probability from 14 discrete values $\{-7, \ldots,-1,1, \ldots, 7\}$. Then $C$ is normalized by its maximum amplitude: $C \mapsto C / \max _{i j}\left(\left|C_{i j}\right|\right)$.

- Dense MAX-CUT graphs: Every upper-triangular element of $C$ is independently chosen with $50 \%$ probability to be either 0 or 1 .

- Cubic MAX-CUT graphs: We sample uniformly from the set of 3-regular graphs, where every vertex has degree 3 . These graphs were generated with the LightGraphs.jl package ${ }^{57}$.

\section{Solving for modulation coefficients}

The problem of finding the matrix of modulation coefficients $F$ is to satisfy $C_{i j} \approx C_{\text {effi } i j}$, as defined in (3). In a fully numerical approach, this problem can be solved by numerically minimizing the objective function

$\sum_{i \neq j}\left(\lambda_{C} C_{i j}-\int_{0}^{2 \pi} J_{i j} \cos \left[(i-j) t+\sum_{k=1}^{N-1}\left(F_{i}^{(k)}-F_{j}^{(k)}\right) \sin k t\right] \frac{\mathrm{d} t}{2 \pi}\right)^{2}$.

For $N<100$, we numerically optimize a relaxed version of this problem (using an iterative approach; see the Supplementary Information), which for these values of $N$ provides low-error solutions.

For $N>100$, the above procedure scales poorly with $N$, so we turn to an approximate approach. If we assume the total phase deviation $\sum_{k=1}^{N-1}\left[F_{i}^{(k)}-F_{j}^{(k)}\right] \sin (k \wedge t) \sim \zeta$ where $\zeta \ll 1$ (i.e., the modulations are small), then the equation $C_{i j}=C_{\text {effij } i j}$ can be expanded to second order in $\zeta$ to obtain the equations

$$
\begin{aligned}
\frac{\lambda_{c} C_{i j}}{J_{i j}}= & -\frac{1}{2}\left(F_{i}^{(i-j)}-F_{j}^{(i-j)}\right)-\frac{1}{8} \sum_{l=1}^{N-1-(i-j)}\left(F_{i}^{(l)}-F_{j}^{(l)}\right)\left(F_{i}^{(i-j+l)}-F_{j}^{(i-j+l)}\right) \\
& -\frac{1}{8} \sum_{l=i-j+1}^{N-1}\left(F_{i}^{(l)}-F_{j}^{(l)}\right)\left(F_{i}^{(l-i+j)}-F_{j}^{(l-i+j)}\right) \\
& +\frac{1}{8} \sum_{l=1}^{i-j-1}\left(F_{i}^{(l)}-F_{j}^{(l)}\right)\left(F_{i}^{(i-j-l)}+F_{j}^{(i-j-l)}\right) .
\end{aligned}
$$

An exact solution to these equations does not necessarily solve $C_{i j}=C_{\text {eff } i j}$, but the errors should be small if $\zeta \ll 1$. Since this small-modulation approximation significantly reduces the nonlinearity of the problem, we utilize this second-order approximation as our definition of the design problem to be solved when $N \geq 100$. To solve the problem in this approximation, we again apply a relaxation to solve these algebraic equations via solving an optimization problem instead, with objective functions (one for each $k=1, \ldots, N-1$ ):

$\sum_{j=1}^{N-k}\left(\frac{\lambda_{c} C_{j}^{(k)}}{J_{j}^{(k)}}+\frac{1}{4}\left(F_{j+k}^{(k)}-F_{j}^{(k)}\right)\left[2+\theta_{2 k}^{N-1}\left(F_{j+k}^{(2 k)}-F_{j}^{(2 k)}\right]\right)+u_{j}^{(k)}\right)^{2}$,

where $\theta_{M}^{N}=1$ iff $N \geq M$ and

$$
\begin{aligned}
u_{j}^{(k)}:= & \frac{1}{8} \sum_{l=1}^{N-1-k}\left(1-\delta_{k, l}\right)\left(F_{j+k}^{(l)}-F_{j}^{(l)}\right)\left(F_{j+k}^{(k+l)}-F_{j}^{(k+l)}\right) \\
& +\frac{1}{8} \sum_{l=k+1}^{N-1}\left(1-\delta_{2 k, l}\right)\left(F_{j+k}^{(l)}-F_{j}^{(l)}\right)\left(F_{j+k}^{(l-k)}-F_{j}^{(l-k)}\right) \\
& -\frac{1}{8} \sum_{l=1}^{k-1}\left(F_{j+k}^{(l)}-F_{j}^{(l)}\right)\left(F_{j+k}^{(k-l)}-F_{j}^{(k-l)}\right) .
\end{aligned}
$$

Our approach is to iteratively optimize these objective functions one at a time, repeating until convergence or after a fixed number of optimizations have been performed. For additional information, see the Supplementary Information.

\section{Quantum simulations}

We perform quantum simulations in this work to analyze the performance of the dynamical-coupling scheme relative to the statically coupled system, 
especially in the presence of dissipation. Our dissipative quantum model is described by the standard Lindblad master equation

$$
\frac{\mathrm{d} \hat{\rho}}{\mathrm{d} t}=-\mathrm{i}[\hat{H}, \hat{\rho}]+\sum_{i}\left(\hat{L}_{i} \hat{\rho} \hat{L}_{i}^{\dagger}-\frac{1}{2} \hat{L}_{i}^{\dagger} \hat{L}_{i} \hat{\rho}-\frac{1}{2} \hat{\rho} \hat{L}_{i}^{\dagger} \hat{L}_{i}\right)
$$

where $\hat{H}$ is the Hamiltonian of the system, and $\hat{L}_{i}$ are the Lindblad operators that describe the effect of dissipation due to coupling to the environment; the time dependence of all operators has been omitted for brevity. For the statically coupled scheme, the Hamiltonian is given by (1) while the Lindblad operators are given by $\hat{L}_{i}=\sqrt{\kappa} \hat{a}_{i}$. For the dynamicalcoupling scheme, the Hamiltonian is given by (2), while the Lindblad operators remain $\hat{L}_{i}=\sqrt{k} \hat{a}_{i}$, since the terms in (8) involving the Lindblad operators are invariant under the overall frame rotations imposed by the dynamical-coupling scheme (see the Supplementary Information for more details).

Directly simulating (8) is difficult due to the large Hilbert-space dimension of the quantum state. To circumvent the memory requirement of storing a full density matrix, we utilize the Monte-Carlo wavefunction method (i.e., quantum-jump method) to solve (8) via stochastic sampling ${ }^{58}$. Numerically, the simulations were performed with the QuTiP library (version 4.3.1) in Python ${ }^{59}$.

Given a spin configuration $\sigma=\left(\sigma_{1}, \ldots, \sigma_{N}\right)$ where $\sigma_{i}= \pm 1$, we can define the probability of the quantum state $\rho(\hat{t})$ being in the specific spin configuration (i.e., configuration probability) $\sigma$ as ${ }^{18}$

$\mathrm{P}_{\sigma}(t):=\prod_{i=1}^{N}\left(\int_{\operatorname{sgn} x_{i}=\sigma_{i}} \mathrm{~d} x_{i}\right)\left\langle x_{1}, \ldots, x_{N}|\rho(\hat{t})| x_{1}, \ldots, x_{N}\right\rangle$.

This definition of the success probability is motivated by the fact that the final state of the JPO system is approximately a superposition of coherent states $\left| \pm a_{i}\right\rangle$ for each JPO, and we interpret these coherent states to encode the Ising spins $\sigma_{i}= \pm 1$.

\section{Classical simulations}

For the statically coupled system, we simulate the following classical EOMs:

$\frac{\mathrm{d} a_{i}}{\mathrm{~d} t}=\mathrm{i}\left(\delta_{i}+X\left|a_{i}\right|^{2}\right) a_{i}-\mathrm{ir} a_{i}^{*}+\mathrm{i} \lambda_{C} \sum_{i \neq j} C_{i j} a_{j}$.

For the dynamically coupled system, we simulate the following classical EOMs:

$$
\begin{aligned}
\frac{\mathrm{d} a_{i}}{\mathrm{~d} t}= & \mathrm{i}\left(\delta_{i}+x\left|a_{i}\right|^{2}\right) a_{i}-\mathrm{i} r a_{i}^{*} \\
& +\mathrm{i} \lambda_{C} \sum_{j \neq i} J_{i j} \exp \left[-\mathrm{i} \wedge(j-i) t+\mathrm{i} \delta \phi_{j}(t)-\mathrm{i} \delta \phi_{i}(t)\right] a_{j} .
\end{aligned}
$$

We simulate both sets of classical EOMs using the ODE solver library DifferentialEquations.jl (version 4.5.0) in Julia ${ }^{60}$. Regarding the initial conditions of the ODE, we follow the approach of Ref. ${ }^{18}$ and sample random initial-field amplitudes $a_{i}(t=0)$, according to $a_{i}(t=0)=\sqrt{n_{0} / 2} \mathrm{e}^{2 \pi i u_{i}} z_{i}$, where $z_{i}$ are iid random variables drawn from the standard normal distribution, while $u_{i}$ are iid random variables drawn from the uniform distribution on the interval $[0,1]$ For all classical simulations, we choose $n_{0}=10^{-2}$. As with the quantum simulations, we simulate the classical EOMs on a finite number of trajectories.

In our classical simulations, we identify the spin configuration of a trajectory $a(t)$ using the relation $\sigma_{i}(t)=\operatorname{sgn}\left(\operatorname{Re} a_{i}(t)\right)$. The spin configurations over the ensemble of trajectories then determine the success probability.

\section{DATA AVAILABILITY}

The data that support the findings of this study are available in Zenodo with the identifier https://doi.org/10.5281/zenodo.3739380

\section{CODE AVAILABILITY}

The source code for running both quantum and semiclassical simulations of the proposed dynamical-coupling scheme can be found at https://doi.org/10.5281/ zenodo.3739380. Further source code used in this study, such as that performing sweeps over parameters when generating the figures, is available from the corresponding author upon reasonable request.
Received: 16 November 2019; Accepted: 15 April 2020;

Published online: 29 May 2020

\section{REFERENCES}

1. Kadowaki, T. \& Nishimori, H. Quantum annealing in the transverse Ising model. Phys. Rev. E 58, 5355-5363 (1998)

2. Farhi, E., Goldstone, J., Gutmann, S. \& Sipser, M. Quantum computation by adiabatic evolution. Preprint at http://arxiv.org/abs/quant-ph/0001106 (2000).

3. Boixo, S. et al. Evidence for quantum annealing with more than one hundred qubits. Nat. Phys. 10, 218-224 (2014).

4. Perdomo-Ortiz, A. et al. Readiness of quantum optimization machines for industrial applications. Phys. Rev. Applied 12, 014004 (2019).

5. Katzgraber, H. G. et al. Viewing vanilla quantum annealing through spin glasses. Quantum Sci. Technol. 3, 030505 (2018).

6. Hamerly, R. et al. Experimental investigation of performance differences between coherent Ising machines and a quantum annealer. Sci. Adv. 5, eaau0823 (2019).

7. Hauke, P., Katzgraber, H. G., Lechner, W., Nishimori, H. \& Oliver, W. D. Perspectives of quantum annealing: methods and implementations. Rep. Prog. Phys. 83, 054401 (2020).

8. Johnson, M. W. et al. Quantum annealing with manufactured spins. Nature $\mathbf{4 7 3}$ 194-198 (2011)

9. Weber, S. et al. Hardware considerations for high-connectivity quantum annealers. Bull. Am. Phys. Soc. https://meetings.aps.org/Meeting/MAR18/Session/A33.8 (2018).

10. Chen, Y. et al. Progress towards a small-scale quantum annealer I: Architecture. Bull. Am. Phys. Soc. https://meetings.aps.org/Meeting/MAR17/Session/B51.4 (2017).

11. Majer, J. et al. Coupling superconducting qubits via a cavity bus. Nature $\mathbf{4 4 9}$, 443-447 (2007).

12. Dicarlo, L. et al. Demonstration of two-qubit algorithms with a superconducting quantum processor. Nature 460, 240-244 (2009).

13. Mariantoni, M. et al. Implementing the quantum von Neumann architecture with superconducting circuits. Science 334, 61-65 (2011).

14. Song, C. et al. 10-Qubit entanglement and parallel logic operations with a superconducting circuit. Phys. Rev. Lett. 119, 180511 (2017).

15. Song, C. et al. Generation of multicomponent atomic Schrödinger cat states of up to 20 qubits. Science 365, 574-577 (2019).

16. Hoppensteadt, F. \& Izhikevich, E. Oscillatory neurocomputers with dynamic connectivity. Phys. Rev. Lett. 82, 2983-2986 (1999).

17. Louisell, W. H., Yariv, A. \& Siegman, A. E. Quantum fluctuations and noise in parametric processes. I. Phys. Rev. 124, 1646-1654 (1961).

18. Goto, H. et al. Bifurcation-based adiabatic quantum computation with a nonlinear oscillator network. Sci. Rep. 6, 21686 (2016).

19. Nigg, S. E., Lörch, N. \& Tiwari, R. P. Robust quantum optimizer with full connectivity. Sci. Adv. 3, e1602273 (2017)

20. Puri, S., Andersen, C. K., Grimsmo, A. L. \& Blais, A. Quantum annealing with all-toall connected nonlinear oscillators. Nat. Commun. 8, 15785 (2017).

21. Bukov, M., D'Alessio, L. \& Polkovnikov, A. Universal high-frequency behavior of periodically driven systems: from dynamical stabilization to Floquet engineering. Adv. Phys. 64, 139-226 (2015).

22. Eckardt, A. \& Anisimovas, E. High-frequency approximation for periodically driven quantum systems from a Floquet-space perspective. New J. Phys. 17, 093039 (2015).

23. Wang, Z., Marandi, A., Wen, K., Byer, R. L. \& Yamamoto, Y. A coherent ising machine based on degenerate optical parametric oscillators. Phys. Rev. A $\mathbf{8 8}$ 063853 (2013).

24. Marandi, A., Wang, Z., Takata, K., Byer, R. L. \& Yamamoto, Y. Network of timemultiplexed optical parametric oscillators as a coherent Ising machine. Nat. Photonics 8, 937-942 (2014).

25. McMahon, P. L. et al. A fully programmable 100-spin coherent Ising machine with all-to-all connections. Science 354, 614-617 (2016).

26. Inagaki, T. et al. A coherent Ising machine for 2000-node optimization problems. Science 354, 603-606 (2016).

27. Arndt, M. \& Hornberger, K. Testing the limits of quantum mechanical superpositions. Nat. Phys. 10, 271-277 (2014).

28. Lifshitz, R. \& Cross, M. C. Response of parametrically driven nonlinear coupled oscillators with application to micromechanical and nanomechanical resonator arrays. Phys. Rev. B 67, 134302 (2003).

29. Goto, H. Universal quantum computation with a nonlinear oscillator network. Phys. Rev. A 93, 050301 (2016).

30. Puri, S., Boutin, S. \& Blais, A. Engineering the quantum states of light in a Kerrnonlinear resonator by two-photon driving. npj Quantum Inf. 3, 18 (2017).

31. Krantz, $P$. et al. Single-shot read-out of a superconducting qubit using a Josephson parametric oscillator. Nat. Commun. 7, 11417 (2016). 
32. Frattini, N. E., Sivak, V. V., Lingenfelter, A., Shankar, S. \& Devoret, M. H. Optimizing the nonlinearity and dissipation of a SNAIL parametric amplifier for dynamic range. Phys. Rev. Appl. 10, 054020 (2018).

33. Wang, Z. et al. Quantum dynamics of a few-photon parametric oscillator. Phys. Rev. X 9, 021049 (2019).

34. Grimm, A. et al. The Kerr-Cat Qubit: Stabilization, Readout, and Gates. Preprint at http://arxiv.org/abs/1907.12131 (2019).

35. Lechner, W., Hauke, P. \& Zoller, P. A quantum annealing architecture with all-to-all connectivity from local interactions. Sci. Adv. 1, e1500838 (2015).

36. Leghtas, Z. et al. Confining the state of light to a quantum manifold by engineered two-photon loss. Science 347, 853-857 (2015).

37. Mirrahimi, M. et al. Dynamically protected cat-qubits: a new paradigm for universal quantum computation. New J. Phys. 16, 045014 (2014).

38. Puri, S. et al. Stabilized cat in a driven nonlinear cavity: a fault-tolerant error syndrome detector. Phys. Rev. X 9, 041009 (2019).

39. Roy, A. \& Devoret, M. Introduction to parametric amplification of quantum signals with Josephson circuits. Comptes Rendus Phys. 17, 740-755 (2016).

40. Reagor, $M$. et al. Demonstration of universal parametric entangling gates on a multi-qubit lattice. Sci. Adv. 4, eaao3603 (2018).

41. Rønnow, T. F. et al. Quantum computing. Defining and detecting quantum speedup. Science 345, 420-424 (2014).

42. Burnett, J. J. et al. Decoherence benchmarking of superconducting qubits. $n p j$ Quantum Inf. 5, 54 (2019).

43. Krantz, P. et al. A quantum engineer's guide to superconducting qubits. Appl. Phys. Rev. 6, 021318 (2019).

44. Lee, N. R. A. et al. Electric fields for light: Propagation of microwave photons along a synthetic dimension. Preprint at http://arxiv.org/abs/1908.10329 (2019).

45. Bunyk, P. I. et al. Architectural considerations in the design of a superconducting quantum annealing processor. IEEE Trans. Appl. Supercond. 24, 1-10 (2014).

46. Oka, T. \& Kitamura, S. Floquet engineering of quantum materials. Annu. Rev. Condens. Matter Phys. 10, 387-408 (2019).

47. Moessner, R. \& Sondhi, S. L. Equilibration and order in quantum Floquet matter. Nat. Phys. 13, 424-428 (2017).

48. Goldman, N., Budich, J. C. \& Zoller, P. Topological quantum matter with ultracold gases in optical lattices. Nat. Phys. 12, 639-645 (2016)

49. Kyriienko, O. \& Sørensen, A. S. Floquet quantum simulation with superconducting qubits. Phys. Rev. Appl. 9, 64029 (2018).

50. Görg, F. et al. Enhancement and sign change of magnetic correlations in a driven quantum many-body system. Nature 553, 481-485 (2018).

51. Gardas, B., Dziarmaga, J., Zurek, W. H. \& Zwolak, M. Defects in quantum computers. Sci. Rep. 8, 4539 (2018)

52. Houck, A. A., Türeci, H. E. \& Koch, J. On-chip quantum simulation with superconducting circuits. Nat. Phys. 8, 292-299 (2012).

53. Roushan, P. et al. Chiral ground-state currents of interacting photons in a synthetic magnetic field. Nat. Phys. 13, 146-151 (2017).

54. Roushan, P. et al. Spectroscopic signatures of localization with interacting photons in superconducting qubits. Science 358, 1175-1179 (2017).

55. Ma, R. et al. A dissipatively stabilized Mott insulator of photons. Nature 566, 51-57 (2019).

56. Yan, Z. et al. Strongly correlated quantum walks with a 12-qubit superconducting processor. Science 364, 753-756 (2019).

57. Bromberger, S. et al. JuliaGraphs/LightGraphs.jl: an optimized graphs package for the Julia programming language, https://doi.org/10.5281/zenodo.889971 (2017).

58. Wiseman, H. M. \& Milburn, G. J. Quantum Measurement and Control (Cambridge University Press, 2009).
59. Johansson, J., Nation, P. \& Nori, F. QuTiP 2: a Python framework for the dynamics of open quantum systems. Comput. Phys. Commun. 184, 1234-1240 (2013).

60. Rackauckas, C. \& Nie, Q. DifferentialEquations.jl - A Performant and Feature-Rich Ecosystem for Solving Differential Equations in Julia. J. Open Res. Softw. 5, 15 (2017).

\section{ACKNOWLEDGEMENTS}

We thank Johannes Majer, Leigh Martin, Tim Menke, Kevin O'Brien, William Oliver, Shruti Puri, Chris Quintana, Shyam Shankar, and Morten Kjaergaard for helpful discussions, and Daniel Wennberg and Ryotatsu Yanagimoto for comments on a draft of the manuscript. We also thank the anonymous reviewers for feedback that strengthened our manuscript. This research was partially funded by NSF award PHY1648807 (T.O., E.N.) and Stanford University (the Nano- and Quantum Science and Engineering Postdoctoral Fellowship; P.L.M.). We also wish to thank NTT Research for their financial and technical support.

\section{AUTHOR CONTRIBUTIONS}

T.O. and E.N. contributed equally to this work. P.L.M. conceived and supervised the project. T.O. and E.N. derived the analytical models, performed the numerical simulations, and produced the figures. P.L.M., T.O., and E.N. wrote the manuscript.

\section{COMPETING INTERESTS}

The authors declare no competing interests.

\section{ADDITIONAL INFORMATION}

Supplementary information is available for this paper at https://doi.org/10.1038/ s41534-020-0279-z.

Correspondence and requests for materials should be addressed to P.L.M.

Reprints and permission information is available at http://www.nature.com/ reprints

Publisher's note Springer Nature remains neutral with regard to jurisdictional claims in published maps and institutional affiliations.

Open Access This article is licensed under a Creative Commons Attribution 4.0 International License, which permits use, sharing, adaptation, distribution and reproduction in any medium or format, as long as you give appropriate credit to the original author(s) and the source, provide a link to the Creative Commons license, and indicate if changes were made. The images or other third party material in this article are included in the article's Creative Commons license, unless indicated otherwise in a credit line to the material. If material is not included in the article's Creative Commons license and your intended use is not permitted by statutory regulation or exceeds the permitted use, you will need to obtain permission directly from the copyright holder. To view a copy of this license, visit http://creativecommons. org/licenses/by/4.0/.

(c) The Author(s) 2020 